\title{
Analytical Model to Profile the Hob Mill for Generating Quadrilobed Pump Rotor
}

\author{
Gabriel Frumuşanu and Nicolae Oancea
}

\begin{abstract}
This paper presents a newly developed analytical model with application in profiling the hob mill for generating a pump rotor having four circular lobes with rectilinear directrix. The analytical model of rotor surface is deducted at first. The generating rack-tool is secondly determined and then, the peripheral surface of the hob mill results as envelop of surfaces family generated by the successive positions of generating rack-tool. The profiling solution lies on the fundamental theorems concerning the reciprocal enwrapped surfaces. A calculus algorithm and a numerical model developed in MatLab are proposed. The characteristic curve of hob mill tooth surface is found out in the case of a given rotor profile.
\end{abstract}

Index Terms-Quadrilobed rotor, analytical model, hob mill profiling, intermediary surface method.

\section{INTRODUCTION}

The generating of ordinate whirls of surfaces (with application, for example, in machining gears or wheels with periodic profile) can be realized by different means, such as milling with profiled tools (disc cutter, end-mill cutter), slotting the blank from two successive teeth, or milling with a hob mill (the most productive solution). It should be also noticed that if using NC machine tools, then it is possible to generate, with reasonable productivity, gear teeth having any type of profile.

The design of hob mill used for generating an ordinate whirl of surfaces - i.e. the teeth to be machined (no matter if cylindrical or helical), requires to profile the tool tooth, because the shape of tool active teeth depends, in definitory manner, on generated profile shape.

The generating process, when using a hob mill in this purpose, is a problem of enwrapping with punctiform contact, taking place between tool helical flank and cylindrical/helical flank belonging to generated whirl of surfaces (gear teeth following to be machined).

There are diverse methods enabling hob mill tooth profiling, such as Olivier second theorem [1], which is a general solution having, sometimes, the inconvenient of requiring to manipulate complicated relations, hence it becomes difficult to apply in practice.

A solution for easier solving the above-mentioned problem, at least in what concerns the complexity of used equations, is the Intermediary Surface Method [1], [2] (here the generating rack-tool being considered as intermediary surface). Applying it supposes to make two successive steps: $i$ ) finding

Manuscript received September 27, 2017; revised March 1, 2018.

G. Frumuşanu is with the Manufacturing Engineering Department, Dunărea de Jos University of Galaţi, Romania (e-mail: gabriel.frumusanu@ ugal.ro). the generating rack-tool profile and $i i$ ) finding the envelop of rack-tool surfaces family, this envelop meaning the primary peripheral surface of the mill hob. Hereby, the enwrapping problem with punctiform contact is transformed in two successive problems of enwrapping with linear contact: the first regards the contact between the generated tooth flank ant the rack-tool flank, while the second - the contact between the rack-tool flank and the tool primary peripheral surface. The method enables to find the characteristic curves in the two successive steps and then, starting from here, the point of contact between the generated surface and tool primary peripheral surface is determined. Gohman general theorem [1-3] may be applied for finding both characteristic curves.

In [3], there is also presented a profiling solution developed on the base of complementary theorems - the Minimum Distance Method or the Substitutive Circles Family Method can be applied, for example.

A very ingenious solution for the same problem has been found by Liuksin [4], lying on the decomposition of the helical motion needed for generating the hob mill peripheral surface.

The development of graphical design media has made possible to approach and to solve (with incontestable benefits) the addressed profiling problem in graphical form - e.g. in [5] the solution was determined by using AutoCAD, while in $[6,7]$ - CATIA.

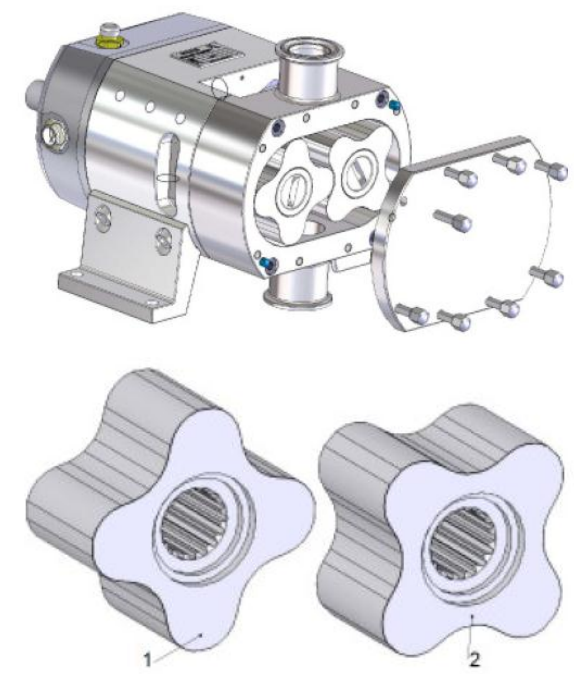

Fig. 1. Quadrilobed rotors pump [8].

The quadrilobed rotors pumps [8], Fig. 1, are increasingly used in recent years due to their functional advantages. However, despite the existence of information concerning their functioning and maintenance, from commercial reasons, nobody tells anything about rotors profile and rotors manufacturing either. 
This paper proposes an analytical algorithm, developed on the base of Intermediary Surface Method, to be applied for profile the hob mill that could be used for machining the quadrilobed rotors. Next section presents the analytical model of the quadrilobed rotor. Third section is about the particular manner of applying the Intermediary Surface Method for profiling the hob mill for quadrilobed rotor. Fourth section deals with a numerical application of the profiling algorithm, while the last one is for conclusion.

\section{ANALYTICAL MODEL OF THE QUADRILOBED ROTOR}

Fig. 2 presents the geometrical parameters (angles and radii) needed to define the quadrilobed rotor profile, in transversal section, referred to $X Y$ local system. Because the rotor has two perpendicular axis of symmetry, it is enough to look at only a quarter of the profile, here denoted by $A B C$. It results by joining two arcs of circle, $A B$ (convex) and $B C$ (concave). The two arcs have the radii $r$ and $r_{l}$, respectively.

The centrode attached to the rotor is a circle of $R_{r}$ radius, having the center in rotor symmetry point, $O$.

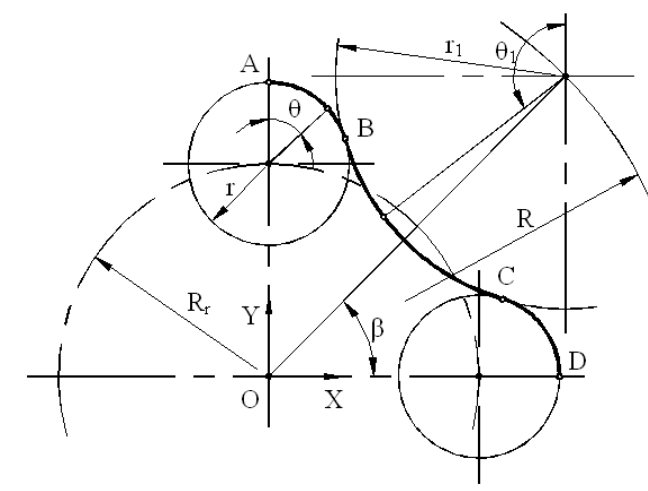

Fig. 2. Quadrilobed rotor geometry.

According to notations from Fig. 2, the equations of the two arcs are:

$$
A B \mid \begin{aligned}
& X=r \sin \theta \\
& Y=R_{r}+r \cos \theta
\end{aligned}
$$

with $\theta \in\left[0, \theta_{M}\right]$, respective

$$
B C \mid \begin{aligned}
& X=R \cos \beta-r_{1} \sin \theta_{1} \\
& Y=R \sin \beta+r_{1} \cos \theta_{1},
\end{aligned}
$$

with $\theta_{1} \in\left[\theta_{1 m}, \pi / 2+\beta\right]$. The limit-values $\theta_{M}$ and $\theta_{1 m}$, measured as shown in Fig. 2, correspond to the point where the two arcs join, namely $B$. Their values result from equations (1) and (2), by imposing the condition that $B$-point simultaneously belongs to both arcs. From symmetry reasons, $\beta=\pi / 4$.

\section{APPLICATION OF INTERMEDIARY SURFACE METHOD}

\section{A. Families of Rotor Flank Surfaces}

Here, the Intermediary Surface Method is directly applied to the addressed profiling problem. This supposes, at the first stage, to determine the surface of the rack-gear associated to the couple of profiles $A B$ and $B C$, which are attached to $C_{l}$ centrode. Fig. 3 presents the ensemble formed by the rolling centrodes: $C_{l}$ (circle of $R_{r}$ radius), belonging to the quadrilobed rortor and $C_{2}-$ the rolling straight line of the generating rack-gear. The parameters characterizing centrodes motions are $\Phi_{1}$ - the angle of rotation around $z$ axis for $C_{1}$, respective $\lambda$ - the distance of translation along $x$ axis, for $C_{2}$. The two centrodes are considered in rolling without slipping motion, which means that:

$$
\lambda=R_{r} \cdot \Phi_{1}
$$

The relation (3) means the condition of rolling between the two centrodes.

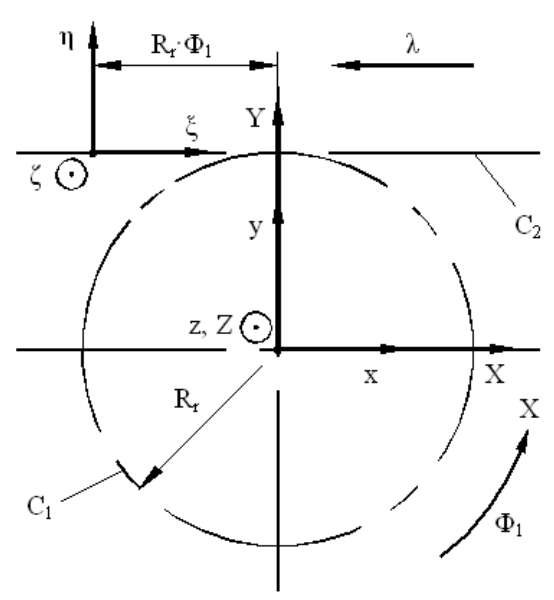

Fig. 3. The centrodes rolling \& reference systems.

In Fig. 3, there are also presented the reference systems needed for determine the equation of the relative motion between the centrodes:

- $\quad x y z$ that is the global system, which is fix

- $X Y Z$ - local system, attached to $\mathrm{C}_{1}$ centrode and executing a rotation of $\Phi_{1}$ angle around z-axis

- $\quad \xi \eta \zeta$ - local system, attached to $\mathrm{C}_{2}$ centrode and executing a translation of $\lambda$ along $\mathrm{x}$ axis.

The generating process kinematics can be defined by starting from the absolute motions equations:

$$
x=\omega_{3}^{T}\left(\Phi_{1}\right) \cdot X
$$

meaning $C_{I}$ rotation (hence rotor generated flanks rotation), with $\omega_{3}-$ the matrix of rotation around z-axis, and

$$
\xi=x-A, \text { with } A=\left(\begin{array}{c}
-R_{r} \cdot \Phi_{1} \\
R_{r} \\
0
\end{array}\right),
$$

meaning $C_{2}$ translation.

The relative motion equation results after elimination of $x$ between equations (4) and (5):

$$
\xi=\omega_{3}{ }^{T}\left(\Phi_{1}\right) \cdot X-\left(\begin{array}{c}
-R_{r} \cdot \Phi_{1} \\
R_{r} \\
0
\end{array}\right) .
$$


Now, if in (6) the matrix $X$ means the composite flank of the rotor to be generated, then, after developing, the equations of rotor family of surfaces will result, as written in the reference system of the future rack-gear. We firstly input in $X$ the equations (1) of $A B$ arc:

$$
\left(\begin{array}{c}
\xi \\
\eta \\
\zeta
\end{array}\right)=\left(\begin{array}{ccc}
\cos \Phi_{1} & -\sin \Phi_{1} & 0 \\
\sin \Phi_{1} & \cos \Phi_{1} & 0 \\
0 & 0 & 1
\end{array}\right) \cdot\left(\begin{array}{c}
r \sin \theta \\
R_{r}+r \cos \theta \\
t
\end{array}\right)-\left(\begin{array}{c}
-R_{r} \cdot \Phi_{1} \\
R_{r} \\
0
\end{array}\right)
$$

where $t$ means the parameter of the cylindrical surface generatrice, along $Z$-axis. After developing, the parametric equations of rotor family of surfaces (corresponding to $\mathrm{AB}$ segment of profile) will result:

$$
\left(\Sigma_{A B}\right)_{\Phi_{1}} \mid \begin{aligned}
& \xi=r \sin \theta \cos \Phi_{1}-\left(R_{r}+r \cos \theta\right) \sin \Phi_{1}+R_{r} \cdot \Phi_{1} \\
& \eta=r \sin \theta \sin \Phi_{1}+\left(R_{r}+r \cos \theta\right) \cos \Phi_{1}-R_{r} ; \\
& \zeta=t .
\end{aligned}
$$

The variable parameters of the surfaces family (8) are: $\theta, t$ and $\Phi_{l}$. The surfaces family corresponding to $B C$ profile segment similarly results as:

$$
\left(\Sigma_{B C}\right)_{\Phi_{1}} \mid \begin{aligned}
\xi= & \left(R \cos \beta-r_{1} \sin \theta_{1}\right) \cos \Phi_{1}- \\
& -\left(R \sin \beta+r_{1} \cos \theta_{1}\right) \sin \Phi_{1}+R_{r} \cdot \Phi_{1} \\
\eta & \left(R \cos \beta-r_{1} \sin \theta_{1}\right) \sin \Phi_{1}+ \\
& +\left(R \sin \beta+r_{1} \cos \theta_{1}\right) \sin \Phi_{1}-R_{r} \\
\zeta= & t
\end{aligned}
$$

\section{B. Generating Rack-gear Profile}

For finding the generating rack-gear profile, the equations representing the surfaces family (8) and (9) should be associated with the specific enveloping condition, which according to Gohman [1-3] has the expression:

$$
\vec{N}\left(\Sigma_{A B / B C}\right) \cdot \vec{R}_{\Phi_{1}}=0
$$

Here $\vec{R}_{\Phi_{1}}$ means the derivative of $\mathrm{X}$ matrix relative to $\Phi_{l}$. The form of $\mathrm{X}$ can be expressed from (6) as

$$
X=\omega_{3}\left(\Phi_{1}\right)\left\lfloor\xi+\left(\begin{array}{c}
-R_{r} \cdot \Phi_{1} \\
R_{r} \\
0
\end{array}\right)\right\rfloor
$$

which leads to:

$$
R_{\Phi_{1}}=\dot{\omega}_{3}\left(\Phi_{1}\right)\left[\omega_{3}{ }^{T}\left(\Phi_{1}\right) \cdot X\right]+\omega_{3}\left(\Phi_{1}\right) \cdot\left(\begin{array}{c}
-R_{r} \\
0 \\
0
\end{array}\right) .
$$

In vector format, (12) is equivalent to:

$$
\vec{R}_{\Phi_{1}}=\left(Y-R_{r} \cos \Phi_{1}\right) \cdot \vec{i}+\left(-X+R_{r} \sin \Phi_{1}\right) \cdot \vec{j}+t \cdot \vec{k}
$$

$\vec{R} \Phi_{1}$ representing, in fact, the speed in the relative motion referred to $X Y Z$ system.
In the distinct cases of the two rotor flank sectors, $A B$ and $B C$ (see Fig. 2), $\vec{R}_{\Phi_{1}}$ takes the forms:

$$
\begin{aligned}
\left(\vec{R}_{\Phi_{1}}\right)_{A B}= & \left(R_{r}+r \cos \theta-R_{r} \cos \Phi_{1}\right) \cdot \vec{i}+ \\
& +\left(-r \sin \theta+R_{r} \sin \Phi_{1}\right) \cdot \vec{j}+t \cdot \vec{k}
\end{aligned}
$$

respective

$$
\begin{aligned}
\left(\vec{R}_{\Phi_{1}}\right)_{B C}= & \left(R \sin \beta+r_{1} \cos \theta_{1}-R_{r} \cos \Phi_{1}\right) \cdot \vec{i}+ \\
& +\left(-R \cos \beta+r_{1} \sin \theta_{1}+R_{r} \sin \Phi_{1}\right) \cdot \vec{j}+t \cdot \vec{k}
\end{aligned}
$$

Regarding the expression of the normal versor to the two rotor flank surfaces, their expressions are:

$$
\vec{N}_{\Sigma_{A B}}=-\sin \theta \cdot \vec{i}-\cos \theta \cdot \vec{j}
$$

and

$$
\vec{N}_{\Sigma_{B C}}=-\sin \theta_{1} \cdot \vec{i}+\cos \theta_{1} \cdot \vec{j}
$$

By replacing in (10) the forms (14) and (16) and making the subsequent calculus, the enveloping conditions for $A B$ flank sector resumes to:

$$
\Phi_{1}=0
$$

For finding the enveloping condition in $B C$ flank sector case, the forms (15) and (17) input in (10) is leading to:

$$
\Phi_{1}=\pi-\arcsin \left[R \cos \left(\theta_{1}-\beta\right) / R_{r}\right]-\theta_{1} .
$$

The generating rack-gear surface can be finally determined by associating (18) with (8), for $A B$ sector and (19) with (9), for $B C$ sector. In first case, surface parameters are $\theta$ and $t$, while in the second $-\theta_{1}$ and $t$.

\section{Mill Hob Profiling}

Once deducted the parametric equations of generating rack-gear, written in $\xi \eta \zeta$ system, the surfaces family generated by the rack-gear, referred to hob mill system, and further, the hob mill primary peripheral surface can be also determined.

In Fig. 5, there are presented the hob mill axis position, relative to generating rack-gear system and the reference systems required for finding the hob mill profile. Hob mill axis is situated in a plane normal to $\eta$-axis, at the distance $a$ from $\eta \zeta$ plane.

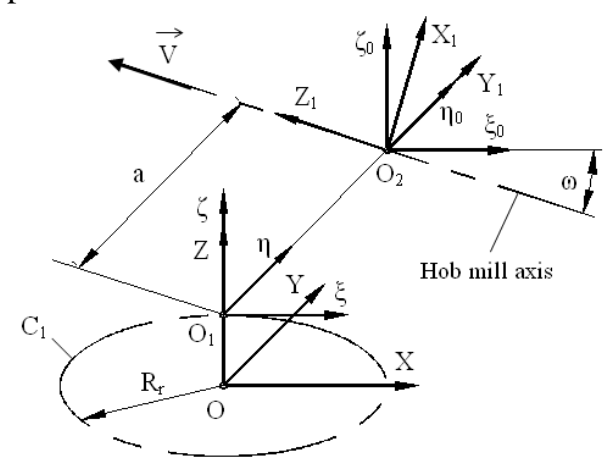

Fig. 4. Hob mill positioning \& reference systems. 
The reference systems newly defined in Fig. 4 are:

- $\xi_{0} \eta_{0} \zeta_{0}-$ local auxiliary system, having its axis parallel to $\xi \eta \zeta$ system ones and the origin in $O_{2}$, onto hob mill axis

- $X_{l} Y_{1} Z_{1}$ - local system, attached to mill hob, $Z_{l}$-axis being collinear with $\vec{V}$ and $X_{l} Z_{l}$ plane parallel to $\xi \zeta$.

The angle between $\eta$-axis and $\vec{V}$ can be calculated with:

$$
\omega=\arcsin \left[R_{r} /(4 \cdot a)\right]
$$

The following co-ordinates transforms can be written:

$$
\left(\begin{array}{l}
\xi_{0} \\
\eta_{0} \\
\zeta_{0}
\end{array}\right)=\left(\begin{array}{l}
\xi \\
\eta \\
\zeta
\end{array}\right)-\left(\begin{array}{l}
0 \\
a \\
0
\end{array}\right)
$$

and

$$
\left(\begin{array}{c}
X_{1} \\
Y_{1} \\
Z_{1}
\end{array}\right)=\left(\begin{array}{ccc}
\sin \omega & 0 & \cos \omega \\
0 & 1 & 0 \\
-\cos \omega & 0 & \sin \omega
\end{array}\right) \cdot\left(\begin{array}{c}
\xi_{0} \\
\eta_{0} \\
\zeta_{0}
\end{array}\right) .
$$

From (21) and (22) finally results:

$$
\mid \begin{aligned}
& X_{1}=\xi \sin \omega+\zeta \cos \omega \\
& Y_{1}=\eta-a \\
& Z_{1}=-\xi \cos \omega+\zeta \sin \omega .
\end{aligned}
$$

The relations (23) enable to transpose the equations of generating rack-gear flanks into hob mill reference system. The distance $a$ from (20) and (21) may be chosen equal to the rolling radius of the hob mill.

Finding of the primary peripheral surface of the hob mill generating the quadrilobed rotor requires at first to give a helical motion of $v$ angle and $p$ parameter, along $\vec{V}$ axis, to generating rack-gear flanks:

$$
X_{1}=\omega_{3}^{T}(v) \cdot X_{1}^{*}+p \cdot v \cdot \vec{k}
$$

where $X_{1}^{*}$ is the matrix formed by generating rack-gear co-ordinates. The specific enveloping condition of surfaces family (24) can be written as:

$$
\left|\begin{array}{lll}
\dot{X}_{1 t} & \dot{Y}_{1 t} & \dot{Z}_{1 t} \\
\dot{X}_{1 \theta} & \dot{Y}_{1 \theta} & \dot{Z}_{1 \theta} \\
\dot{X}_{1 v} & \dot{Y}_{1 v} & \dot{Z}_{1 v}
\end{array}\right|=0 .
$$

The couple of relations (24) and (25) determine the profile of the characteristic curve of hob mill peripheral surface.

\section{NUMERICAL APPLICATION}

In order to validate the newly immagined analytical model for profiling the mill hob generating the quadrilobed rotor, we further present a numerical simulation, developed with the help of a MatLab application dedicated to this purpose.

The input data for the results presented below are:

- The radii of arcs composing the quadrilobed rotor: $r=10 \mathrm{~mm}, r_{l}=40 \mathrm{~mm}$
- The quadrilobed rotor's rolling radius: $R_{r}=40 \mathrm{~mm}$

- The mill hob's rolling radius: $R_{r s}=a=50 \mathrm{~mm}$.

The value of $\omega$ angle, calculated with (20), is $0.2014 \mathrm{rad}$. The value of $p$ helical parameter can be determined with:

$$
p=R_{r} /(4 \cdot \cos \omega),
$$

and results of $10.206 \mathrm{~mm}$.

The value of $R$ (from (2) and Fig. 2) and the limit-values for $\theta_{M}$ and $\theta_{l m}$ can be determined by applying elementary geometry theorems and numerical methods [9]. In the addressed profiling example, $R$ results of $69.515 \mathrm{~mm}$, $\theta_{M}=1.3824 \mathrm{rad}$, and $\theta_{l m}=1.7539 \mathrm{rad}$.

The entire profile of quadrilobed rotor, obtained for the mentioned input data by applying (1) and (2) and then by drawing $A C$ profile symmetric curves relative to bisector lines and to co-ordinates axis, is depicted in Fig. 5.

The generating rack-gear profile, determined with (8), (9), (18) and (19) for the considered input data is shown in Fig. 6.

The characteristic curve of the hob mill for generating the quadrilobed rotor has been found through the co-ordinates of 100 points for each of the sectors corresponding to $A B$ and $B C$ profile segments of the rotor. These co-ordinates are sampled in Table 1 and 2, respectively. The 3-D profile of characteristic curve is depicted in Fig. 7.

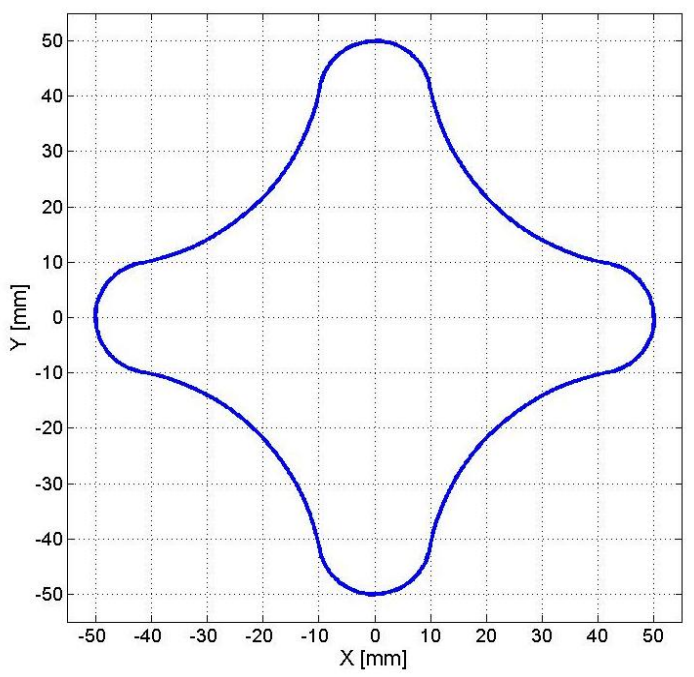

Fig. 5. Quadrilobed rotor profile

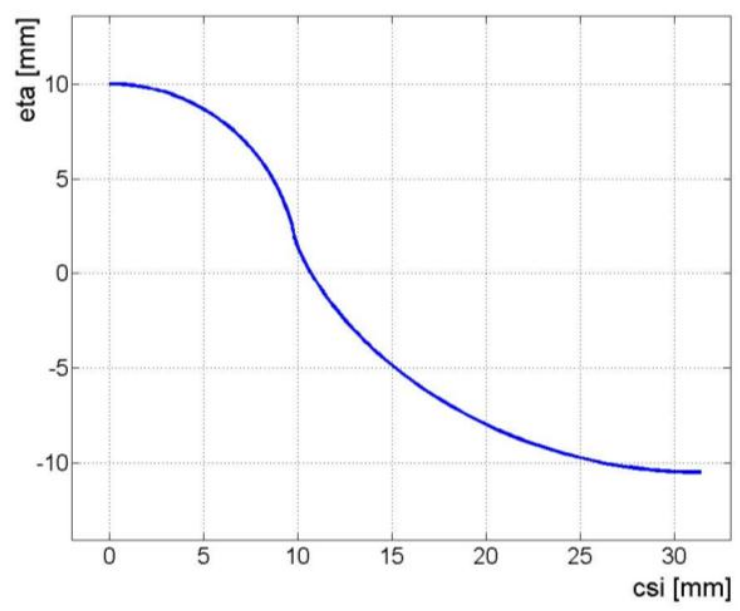

Fig. 6. Generating rack-gear profile. 
TABLE I: THE CHARACTERISTIC CURVE - $A B$ ROTOR SECTOR

\begin{tabular}{cccc}
\hline Point crt. no. & $\mathrm{X}_{1}[\mathrm{~mm}]$ & $\mathrm{Y}_{1}[\mathrm{~mm}]$ & $\mathrm{Z}_{1}[\mathrm{~mm}]$ \\
\hline \hline 1 & -20.000 & -34.641 & -5.343 \\
\hline 2 & -19.976 & -34.655 & -5.480 \\
\hline 3 & -19.953 & -34.672 & -5.617 \\
\hline 4 & -19.932 & -34.690 & -5.754 \\
\hline 5 & -19.911 & -34.710 & -5.890 \\
\hline 48 & -19.981 & -37.050 & -11.322 \\
\hline 49 & -20.005 & -37.135 & -11.430 \\
\hline 50 & -20.030 & -37.222 & -11.536 \\
\hline 51 & -20.056 & -37.310 & -11.642 \\
\hline 52 & -20.083 & -37.399 & -11.746 \\
\hline & $\ldots \ldots \ldots$ & $\ldots \ldots$ \\
\hline 96 & -22.110 & -42.176 & -14.850 \\
\hline 97 & -22.172 & -42.297 & -14.883 \\
\hline 98 & -22.234 & -42.418 & -14.913 \\
\hline 99 & -22.298 & -42.539 & -14.941 \\
\hline 100 & -22.361 & -42.661 & -14.968 \\
\hline \hline
\end{tabular}

TABLE II: THE CHARACTERISTIC CURVE - BC ROTOR SECTOR

\begin{tabular}{cccc}
\hline \hline Point crt. no. & $\mathrm{X}_{1}[\mathrm{~mm}]$ & $\mathrm{Y}_{1}[\mathrm{~mm}]$ & $\mathrm{Z}_{1}[\mathrm{~mm}]$ \\
\hline 1 & -22.344 & -42.758 & -14.969 \\
\hline 2 & -22.554 & -42.497 & -15.009 \\
\hline 3 & -22.764 & -42.364 & -15.057 \\
\hline 4 & -22.972 & -42.306 & -15.115 \\
\hline 5 & -23.177 & -42.298 & -15.183 \\
\hline & $\ldots \ldots \ldots \ldots$ & $\ldots \ldots$ \\
\hline 48 & -28.899 & -48.415 & -22.984 \\
\hline 49 & -28.975 & -48.550 & -23.231 \\
\hline 50 & -29.048 & -48.682 & -23.481 \\
\hline 51 & -29.119 & -48.813 & -23.732 \\
\hline 52 & -29.188 & -48.942 & -23.985 \\
\hline$\ldots 6$ & -30.306 & -52.315 & -36.240 \\
\hline 97 & -30.293 & -52.336 & -36.532 \\
\hline 98 & -30.277 & -52.353 & -36.823 \\
\hline 99 & -30.260 & -52.368 & -37.115 \\
\hline 100 & -30.242 & -52.381 & -37.407 \\
\hline \hline
\end{tabular}

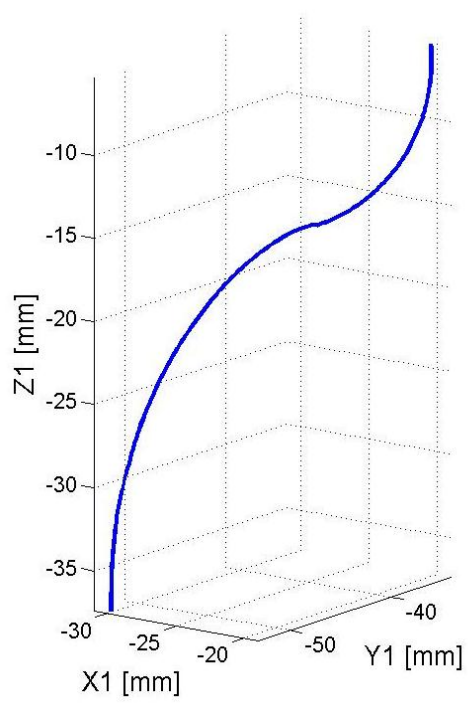

Fig. 7. Mill hob's characteristic curve.

\section{CONCLUSION}

This paper presents a new analytical model to profile the hob mill for generating the quadrilobed pump rotor, developed on the base of the Intermediary Surface Method here the intermediary surface being the generating rack-gear. The addressed profile of quadrilobed rotor is composite, formed by two arcs of circle.

The presented profiling algorithm proves to be relatively easy to apply, in the case when the surfaces to generate have simple analytical curves as profile. The profiling algorithm can be also used in the other situations, after substituting the generated surfaces profile by substitutive curves - e.g. by Bezier polynomial functions having of inferior degree.

The numerical application, developed with the help of a dedicated soft product written in MatLab, sustains the feasibility of the suggested profiling algorithm and proves its high accuracy and efficiency.

\section{REFERENCES}

[1] F. L. Litvin, Theory of gearing, NASA, Scientific and Technical information Division, Washington DC, 1984.

[2] S. P. Radzevich, Kinematics Geometry of Surface Machining, CRC Press, London, 2008.

[3] N. Oancea, Surfaces Generation through Winding, vol. I - III, Galati University Press, Galaţi, 2004.

[4] V. S Lyucshin, Theory of Helical Surfaces for Designing Cutting Tools, Mashinostroenye, Moscow, 1968.

[5] I. Baicu and N. Oancea, Cutting Tools Profiling by Solid Modelling, Tehnica Info Publishing House, Chişinău, 2002.

[6] V. Berbinschi, V. G. Teodor, and N. Oancea, "3D graphical method for profiling tools that generate helical surfaces," Int. Journal of Advanced Manufacturing Technology, vol. 60, 2012, pp. 505-512.

[7] V.G. Teodor, N. Baroiu, S. Berbinschi and N. Oancea, "A graphical expression for the method of substitutive circles family applied for profiling side mill designed to generate sealing worms of cycloid pumps," Applied Mechanics and Materials vol. 809-810, 2015, pp. 998-1003.

[8] Operation and Maintenance Manual C and CF Series, Lobe Positive Displacement Group, Rev. 4 del 01/01/2010, OMAC s.r.l., Rubiera, Italy.

[9] G. Frumuşanu, Numerical methods in Technological Engineering, Cartea Universitară Publishing House, Bucharest, 2004.

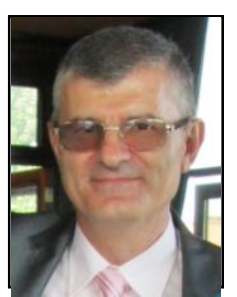

Gabriel Frumuşanu was born in Galaţi, Romania, in 1964. He received his the bachelor in 1988 , the $\mathrm{PhD}$ in 1999, the Habilitation in 2016 in industrial engineering at "Dunărea de Jos" University of Galaţi, Romania.

He is currently professor at "Dunărea de Jos" University of Galaţi, Romania, in the Manufacturing Engineering Department. He published over 150 scientific articles, some of them in prestigious journals (The Internatinal Journal of Advanced Manufacturing Technology, Indian Journal of Engineering and Material Sciences, International Journal of Mechanics, Materials and Manufacturing). He owns 3 patents. He participated at numerous International conferences (Spain, Hungary, Tunisia, Israel, Moldova and Romania). Research interests in machining systems control, cutting tools profiling and environmental impact of the manufacturing process.

Prof. Frumuşanu is member of UASTRO, of editorial boards - Journal of Control and Systems Engineering (JCSE, Bowen Publishing), Proceedings in Manufacturing Systems journal (Romanian Academy), The Annals of „Dunărea de Jos” University, Fascicle V and is scientific reviewer for prestigious ISI Journals (Proceedings of the Institution of Mechanical Engineers, Part B: Journal of Engineering Manufacture, Proceedings of the Institution of Mechanical Engineers, Part C: Journal of Mechanical Engineering Science, Journal of Mechanical Science and Technology). 